\title{
Generalized Fractional Integral Operators Involving Mittag-Leffler Function
}

\author{
Hafte Amsalu (iD and D. L. Suthar (iD \\ Department of Mathematics, Wollo University, P.O. Box 1145, Dessie, Ethiopia \\ Correspondence should be addressed to Hafte Amsalu; yohanahafte@gmail.com \\ Received 2 March 2018; Accepted 11 April 2018; Published 3 June 2018 \\ Academic Editor: Khalil Ezzinbi
}

Copyright (c) 2018 Hafte Amsalu and D. L. Suthar. This is an open access article distributed under the Creative Commons Attribution License, which permits unrestricted use, distribution, and reproduction in any medium, provided the original work is properly cited.

The aim of this paper is to study various properties of Mittag-Leffler (M-L) function. Here we establish two theorems which give the image of this M-L function under the generalized fractional integral operators involving Fox's $H$-function as kernel. Corresponding assertions in terms of Euler, Mellin, Laplace, Whittaker, and $K$-transforms are also presented. On account of general nature of M-L function a number of results involving special functions can be obtained merely by giving particular values for the parameters.

\section{Introduction and Preliminaries}

M-L Function. In 1903, Mittag-Leffler [1] introduced the function $E_{\lambda}(z)$, defined by

$$
E_{\lambda}(z)=\sum_{n=0}^{\infty} \frac{1}{\Gamma(\lambda n+1)} z^{n} \quad(\lambda \in \mathbb{C}) ; \mathfrak{R}(\lambda)>0 .
$$

A further, two-index generalization of this function was given by Wiman [2] as

$$
E_{\lambda, \beta}(z)=\sum_{n=0}^{\infty} \frac{1}{\Gamma(\lambda n+\beta)} z^{n} \quad(\lambda, \beta \in \mathbb{C}),
$$

where $\mathfrak{R}(\lambda)>0$ and $\mathfrak{R}(\beta)>0$.

By means of the series representation a generalization of $\mathrm{M}-\mathrm{L}$ function (2) is introduced by Prabhakar [3] as

$$
E_{\lambda, \beta}^{\gamma}(z)=\sum_{n=0}^{\infty} \frac{(\gamma)_{n}}{\Gamma(\lambda n+\beta) n !} z^{n}
$$

where $\lambda, \beta, \gamma \in \mathbb{C}(\Re(\lambda)>0)$. Further, it is an entire function of order $[\Re(\lambda)]^{-1}$.

Generalized Fractional Integral Operator. Now, we recall the definition of generalized fractional integral operators involving Fox's $H$-function as kernel, defined by Saxena and Kumbhat [4] means of the following equations:

$$
\begin{gathered}
R_{x, r}^{\mu, \alpha}[f(x)]=r x^{-\mu-r \alpha-1} \int_{0}^{x} t^{\mu}\left(x^{r}-t^{r}\right)^{\alpha} \\
\cdot H_{p, q}^{m, n}\left[\begin{array}{c}
\left(a_{p}, A_{p}\right) \\
\left(b_{q}, B_{q}\right)
\end{array}\right] f(t) d t, \\
K_{x, r}^{\varepsilon, \alpha}[f(x)]=r x^{\varepsilon} \int_{x}^{\infty} t^{-\varepsilon-r \alpha-1}\left(t^{r}-x^{r}\right)^{\alpha} \\
\cdot H_{p, q}^{m, n}\left[\begin{array}{c}
\left(a_{p}, A_{p}\right) \\
\left.k V \mid \begin{array}{l}
\left(b_{q}, B_{q}\right)
\end{array}\right] f(t) d t,
\end{array}\right.
\end{gathered}
$$

where $U$ and $V$ represent the expressions

$$
\begin{aligned}
& \left(\frac{t^{r}}{x^{r}}\right)^{\tau}\left(1-\frac{t^{r}}{x^{r}}\right)^{v}, \\
& \left(\frac{x^{r}}{t^{r}}\right)^{\tau}\left(1-\frac{x^{r}}{t^{r}}\right)^{v},
\end{aligned}
$$

respectively, with $\tau, v>0$. The sufficient conditions of operators are given below:

(i) $1 \leq p, q<\infty, p^{-1}+q^{-1}=1$; 
(ii) $\mathfrak{R}\left(\mu+r \tau\left(b_{j} / B_{j}\right)\right)>-q^{-1} ; \mathfrak{R}\left(\alpha+r v\left(b_{j} / B_{j}\right)\right)>-q^{-1}$; $\mathfrak{R}\left(\varepsilon+\alpha+r \tau\left(b_{j} / B_{j}\right)\right)>-p^{-1},(j=1, \ldots, m) ;$

(iii) $f(x) \in L_{P}(0, \infty)$;

(iv) $|\arg k|<\lambda \pi / 2, \lambda>0$, where $\lambda=\sum_{j=1}^{m} B_{j}-\sum_{j=m+1}^{q} B_{j}+\sum_{j=1}^{n} A_{j}-\sum_{j=n+1}^{p} A_{j}>$ 0 .

An interest in the study of the fractional calculus associated with the Mittag-Leffler function and $H$-function, its application in the form of differential, and integral equations of, in particular, fractional orders (see [5-10]).

$H$-Function. Symbol $H_{p, q}^{m, n}(x)$ stands for well known Fox $H$ function [11], in operator (4) and (5) defined in terms of Mellin-Barnes type contour integral as follows:

$$
H_{p, q}^{m, n}(z)=H_{p, q}^{m, n}\left[\begin{array}{c}
\left(a_{p}, A_{p}\right) \\
z \mid \\
\left(b_{q}, B_{q}\right)
\end{array}\right]=\frac{1}{2 \pi i} \int_{L} \chi(s) z^{s} d s
$$

where

$$
\chi(s)=\frac{\prod_{j=1}^{m} \Gamma\left(b_{j}+B_{j} s\right) \prod_{i=1}^{n} \Gamma\left(1-a_{i}-A_{i} s\right)}{\prod_{i=n+1}^{p} \Gamma\left(a_{i}+A_{i} s\right) \prod_{j=m+1}^{q} \Gamma\left(1-b_{j}-B_{j} s\right)},
$$

$m, n, p, q \in \mathbb{N}_{0}$ with $1 \leq m \leq q, 0 \leq n \leq p, A_{i}, B_{j} \in \mathbb{R}_{+}$, $a_{i}, b_{j} \in \mathbb{R}$, or $\mathbb{C}, i=1,2, \ldots, p ; j=1,2, \ldots, q$ such that $A_{i}\left(b_{j}+\right.$ $k) \neq B_{j}\left(a_{i}-l-1\right)\left(k, l \in N_{0} ; i=1,2, \ldots, n ; j=1,2, \ldots, m\right)$.

For the conditions of analytically continuations together with the convergence conditions of $H$-function, one can see $[12,13]$. Throughout the present paper, we assume that these conditions are satisfied by the function.

\section{Images of M-L Function Involving the Generalized Fractional Integral Operators}

In this section, we consider two generalized fractional integral operators involving the Fox's $H$-function as the kernels and derived the following theorems.

Theorem 1. Let $\lambda, \beta, \vartheta, \gamma \in \mathbb{C}, x>0, \mathfrak{R}(\lambda)>0, \mathfrak{R}(\vartheta)>0$, $f(x) \in L_{P}(0, \infty), 1 \leq p \leq 2,|\arg k|<\lambda \pi / 2, \lambda>0, a \in$ $\mathbb{C}$; then the fractional integration $R_{x, r}^{\mu, \alpha}$ of the product of $M-L$ function exists, under the condition

$$
\begin{gathered}
p^{-1}+q^{-1}=1 ; \\
\Re\left(\mu+r \tau\left(\frac{b_{j}}{B_{j}}\right)\right)>-q^{-1} ; \\
\mathfrak{R}\left(\alpha+r v\left(\frac{b_{j}}{B_{j}}\right)\right)>-q^{-1} ;
\end{gathered}
$$

then there holds the following formula:

$$
\begin{aligned}
& R_{x, r}^{\mu, \alpha}\left(t^{\vartheta-1} E_{\lambda, \beta}^{\gamma}\left(a t^{\nu}\right)\right)(x) \\
& =x^{\vartheta-1} \sum_{n=0}^{\infty} \frac{(\gamma)_{n}}{\Gamma(\lambda n+\beta) n !}\left(a x^{\nu}\right)^{n} \times H_{p+2, q+1}^{m, n+2}[k \mid \\
& \left.\left(a_{p}, A_{p}\right),\left(1-\frac{(\mu+\vartheta+1+\nu n)}{r}, \tau\right),(-\alpha, v)\right] \\
& \left.\quad\left(-\frac{(\mu+\vartheta+1+\nu n)}{r}-\alpha, \tau+v\right),\left(b_{q}, B_{q}\right)\right] .
\end{aligned}
$$

Proof. Let $\ell$ be the left-hand side of (10); using (3) and (4), we have

$$
\begin{aligned}
\ell= & r x^{-\mu-r \alpha-1} \int_{0}^{x} t^{\mu+9-1}\left(x^{r}-t^{r}\right)^{\alpha} \\
& \cdot \frac{1}{2 \pi i} \int_{L} \chi(s)(k U)^{s} d s \frac{(\gamma)_{n}}{\Gamma(\lambda n+\beta) n !}\left(a x^{\nu}\right)^{n} d t .
\end{aligned}
$$

Changing the order of the integration valid under the condition given with the theorem, we obtain

$$
\begin{aligned}
\ell= & r x^{-\mu-r \alpha-1} \sum_{n=0}^{\infty} \frac{(\gamma)_{n} a^{n}}{\Gamma(\lambda n+\beta) n !} \times \frac{1}{2 \pi i} \int_{L} \chi(s) \\
& \cdot k^{s} x^{r \alpha-r \tau s}\left\{\int_{0}^{x} t^{\mu+9+v n+r \tau s-1}\left(1-\frac{t^{r}}{x^{r}}\right)^{\alpha+v s} d t\right\} d s .
\end{aligned}
$$

Let the substitution $t^{r} / x^{r}=w$; then $t=x w^{(1 / r)}$ in the above term; we get

$$
\begin{aligned}
& =x^{\vartheta-1} \sum_{n=0}^{\infty} \frac{(\gamma)_{n} a^{n}}{\Gamma(\lambda n+\beta) n !} \frac{x^{v n}}{2 \pi i} \int_{L} \chi(s) k^{s} x^{\nu s} \\
& \quad \times\left\{\int_{0}^{1} w^{(1 / r)(\mu+\vartheta+v n+r \tau s)-1}(1-w)^{\alpha+v s} d w\right\} d s .
\end{aligned}
$$

Using beta function for (13), the inner integral reduces to

$$
\begin{gathered}
=x^{\vartheta-1} \sum_{n=0}^{\infty} \frac{(\gamma)_{n}}{\Gamma(\lambda n+\beta) n !}\left(a x^{\nu}\right)^{n} \frac{1}{2 \pi i} \int_{L} \chi(s) k^{s} \\
\quad \times \frac{\Gamma(((\mu+\vartheta+\nu n) / r)+\tau s) \Gamma(\alpha+1+v s)}{\Gamma(((\mu+\vartheta+\nu n) / r)+\alpha+1+(\tau+v) s)} d s .
\end{gathered}
$$

Interpreting the right-hand side of (14), in view of the definition (7), we arrive at the result (10).

Theorem 2. Let $\lambda, \beta, \vartheta, \gamma \in \mathbb{C}, x>0, \mathfrak{R}(\lambda)>0, \mathfrak{R}(\vartheta)<1$, $f(x) \in L_{P}(0, \infty), 1 \leq p \leq 2,|\arg k|<\lambda \pi / 2, \lambda>0$, and 
$a \in \mathbb{C}$; then the fractional integration $K_{x, r}^{\varepsilon, \alpha}$ of the product of $M-L$ function exists, under the condition

$$
\begin{gathered}
p^{-1}+q^{-1}=1, \\
\Re\left(\alpha+r v\left(\frac{b_{j}}{B_{j}}\right)\right)>-q^{-1}, \\
\Re\left(\varepsilon+\alpha+r \tau\left(\frac{b_{j}}{B_{j}}\right)\right)>-p^{-1}
\end{gathered}
$$

and then the following formula holds:

$$
\begin{aligned}
K_{x, r}^{\varepsilon, \alpha}\left(t^{-\vartheta} E_{\lambda, \beta}^{\gamma}\left(a t^{-v}\right)\right)(x) & \\
= & \left.x^{-\vartheta} \sum_{n=0}^{\infty} \frac{(\gamma)_{n}}{\Gamma(\lambda n+\beta) n !}\left(a x^{-v}\right)^{n} \times H_{p+2, q+1}^{m, n+2}\right] k \mid \\
& \left.\left(a_{p}, A_{p}\right),\left(1-\frac{(\varepsilon+\vartheta+\nu n)}{r}, \tau\right),(-\alpha, v)\right] \\
& \left.\left(-\alpha-\frac{(\varepsilon+\vartheta+\nu n)}{r}, \tau+v\right),\left(b_{q}, B_{q}\right)\right] .
\end{aligned}
$$

Proof. Let $\wp$ be the left-hand side of (16); using (3) and (5), we have

$$
\begin{gathered}
\wp=r x^{\varepsilon} \int_{x}^{\infty} t^{-\varepsilon-9-r \alpha-1}\left(t^{r}-x^{r}\right)^{\alpha} \\
\times \frac{1}{2 \pi i} \int_{L} \chi(s)(k V)^{-s} d s \sum_{n=0}^{\infty} \frac{(\gamma)_{n}}{\Gamma(\lambda n+\beta) n !}\left(a x^{-v}\right)^{n} d t
\end{gathered}
$$

Changing the order of the integration valid under the condition given with the theorem statement, we obtain

$$
\begin{aligned}
\wp= & r x^{\varepsilon} \sum_{n=0}^{\infty} \frac{(\gamma)_{n} a^{n}}{\Gamma(\lambda n+\beta) n !} \frac{1}{2 \pi i} \int_{L} \chi(s) k^{-s} x^{-r \tau \mathrm{s}} \\
& \times\left\{\int_{x}^{\infty} t^{-\varepsilon-9-\nu n+r \tau s-1}\left(1-\frac{x^{r}}{t^{r}}\right)^{\alpha-v s} d t\right\} d s .
\end{aligned}
$$

Letting the substitution $x^{r} / t^{r}=u$, then $t=x / u^{(1 / r)}$ in the above term and, using beta function, we get

$$
\begin{gathered}
=x^{-\vartheta} \sum_{n=0}^{\infty} \frac{(\gamma)_{n}}{\Gamma(\lambda n+\beta) n !}\left(a x^{-v}\right)^{n} \frac{1}{2 \pi i} \int_{L} \chi(s) k^{-s} \\
\quad \times \frac{\Gamma(((\varepsilon+\vartheta+\nu n) / r)-\tau s) \Gamma(\alpha+1-v s)}{\Gamma(((\varepsilon+\vartheta+\nu n) / r)+\alpha+1-(\tau+v) s)} d s .
\end{gathered}
$$

Interpreting the right-hand side of (19), in view of definition (7), we arrive at the result (16).

\section{Integral Transforms of Fractional Integral Involving M-L Function}

In this section, Mellin, Laplace, Euler, Whittaker, and $K$ transforms of the results established in Theorems 1 and 2 have been obtained.

Euler Transform (Sneddon [14]). The Euler transform of a function $f(t)$ is defined as

$$
\begin{aligned}
B\{f(t) ; a, b\}=\int_{0}^{1} t^{a-1}(1-t)^{b-1} f(t) d t, \\
\quad a, b \in \mathbb{C}, \mathfrak{R}(a)>0, \mathfrak{R}(b)>0 .
\end{aligned}
$$

Theorem 3. Let $\lambda, \beta, \vartheta, \gamma, c, d \in \mathbb{C}, \mathfrak{R}(c)>0, \mathfrak{R}(d)>0$, $\mathfrak{R}(\vartheta)>0, \mathfrak{R}(\lambda)>0, p^{-1}+q^{-1}=1 ; f(x) \in L_{P}(0, \infty), 1 \leq p \leq$ $2,|\arg k|<\lambda \pi / 2, \lambda>0, p^{-1}+q^{-1}=1 ; \Re\left(\mu+r \tau\left(b_{j} / B_{j}\right)\right)>$ $-q^{-1} ; \mathfrak{R}\left(\alpha+r v\left(b_{j} / B_{j}\right)\right)>-q^{-1} ;(j=1, \ldots, m)$; then

$$
\begin{aligned}
& B\left\{R_{x, r}^{\mu, \alpha}\left(t^{\vartheta-1} E_{\lambda, \beta}^{\gamma}\left(a t^{\nu}\right)\right) ; c, d\right\}=\Gamma(d) \\
& \quad \sum_{n=0}^{\infty} \frac{(\gamma)_{n}\left(a^{n}\right)}{\Gamma(\lambda n+\beta) n !} \frac{\Gamma(c+\vartheta-1+\nu n)}{\Gamma(c+d+\vartheta-1+v n)} \\
& \quad \times H_{p+2, q+1}^{m, n+2}[k \mid \\
& \left.\quad\left(a_{p}, A_{p}\right),\left(1-\frac{(\mu+\vartheta+1+\nu n)}{r}, \tau\right),(-\alpha, v)\right] \\
& \left.\quad\left(-\frac{(\mu+\vartheta+1+\nu n)}{r}-\alpha, \tau+v\right),\left(b_{q}, B_{q}\right)\right]
\end{aligned}
$$

Proof. Using (10) and (20) gives

$$
B\left\{R_{x, r}^{\mu, \alpha}\left(t^{9-1} E_{\lambda, \beta}^{\gamma}\left(a t^{\nu}\right)\right) ; c, d\right\}=\sum_{n=0}^{\infty} \frac{(\gamma)_{n}}{\Gamma(\lambda n+\beta)} \frac{(a)^{n}}{n !}
$$

$$
\begin{aligned}
& \times H_{p+2, q+1}^{m, n+2}[k \mid \\
& \left.\left(a_{p}, A_{p}\right),\left(1-\frac{(\mu+\vartheta+1+\nu n)}{r}, \tau\right),(-\alpha, v)\right] \\
& \left.\left(-\frac{(\mu+\vartheta+1+\nu n)}{r}-\alpha, \tau+v\right),\left(b_{q}, B_{q}\right)\right] \\
& \times \int_{0}^{1} t^{c+\vartheta+v n-1-1}(1-t)^{d-1} d t
\end{aligned}
$$




$$
\begin{aligned}
= & \sum_{n=0}^{\infty} \frac{(\gamma)_{n}}{\Gamma(\lambda n+\beta)} \frac{(a)^{n}}{n !} \frac{\Gamma(c+\vartheta+\nu n-1) \Gamma(d)}{\Gamma(c+d+\vartheta+\nu n-1)} \\
& \times H_{p+2, q+1}^{m, n+2}[k \mid \\
& \left.\left(a_{p}, A_{p}\right),\left(1-\frac{(\mu+\vartheta+1+\nu n)}{r}, \tau\right),(-\alpha, v)\right] \\
& \left.\left(-\frac{(\mu+\vartheta+1+\nu n)}{r}-\alpha, \tau+v\right),\left(b_{q}, B_{q}\right)\right] .
\end{aligned}
$$

Now, we obtain the result (23). This completes the proof of the theorem.

Theorem 4. Let $\lambda, \beta, \vartheta, \gamma, c, d \in \mathbb{C}, a>0, \mathfrak{R}(c)>0, \mathfrak{R}(d)>$ $0, \mathfrak{R}(\lambda)>0, \mathfrak{R}(1-\vartheta)<1, p^{-1}+q^{-1}=1 ; f(x) \in L_{P}(0, \infty)$, $1 \leq p \leq 2,|\arg k|<\lambda \pi / 2, \lambda>0, p^{-1}+q^{-1}=1 ; \mathfrak{R}(\varepsilon+\alpha+$ $\left.r \tau\left(b_{j} / B_{j}\right)\right)>-p^{-1} ; \mathfrak{R}\left(\alpha+r v\left(b_{j} / B_{j}\right)\right)>-q^{-1} ;(j=1, \ldots, m)$; then

$$
\begin{aligned}
& B\left\{K_{x, r}^{\varepsilon, \alpha}\left(t^{-\vartheta} E_{\lambda, \beta}^{\gamma}\left(a t^{-v}\right)\right) ; c, d\right\}=\Gamma(d) \\
& \cdot \sum_{n=0}^{\infty} \frac{(\gamma)_{n}}{\Gamma(\lambda n+\beta) n !}\left(a^{n}\right) \times \frac{\Gamma(c-\vartheta-v n)}{\Gamma(c+d-\vartheta-v n)} \\
& \cdot H_{p+2, q+1}^{m, n+2}[k \mid \\
& \left.\left(a_{p}, A_{p}\right),\left(1-\frac{(\varepsilon+\vartheta+\nu n)}{r}, \tau\right),(-\alpha, v)\right] \\
& \left.\left(-\alpha-\frac{(\varepsilon+\vartheta+\nu n)}{r}, \tau+v\right),\left(b_{q}, B_{q}\right)\right] .
\end{aligned}
$$

Proof. In similar manner, in proof of Theorem 3, we obtain the result (24).

Mellin Transform (Debnath and Bhatta [15]). The Mellin transform of a function $f(t)$ is defined as

$$
M\{f(t)\}(s)=\int_{0}^{\infty} t^{s-1} f(t) d t, \quad \Re(s)>0 .
$$

Theorem 5. All conditions follow from that stated in Theorem 1 with $\mathfrak{R}(s)>\mathfrak{R}(\nu)$; the following result holds:

$$
M\left\{R_{x, r}^{\mu, \alpha}\left(t^{9-1} E_{\lambda, \beta}^{\gamma}\left(a t^{\nu}\right)\right)\right\}(s)=\sum_{n=0}^{\infty} \frac{(\gamma)_{n}}{\Gamma(\lambda n+\beta) n !}\left(a^{n}\right)
$$

$$
\begin{aligned}
& \times H_{p+2, q+1}^{m, n+2}[k \mid \\
& \left.\left(a_{p}, A_{p}\right),\left(1-\frac{(\mu+\vartheta+1+\nu n)}{r}, \tau\right),(-\alpha, v)\right] \\
& \left.\left(-\frac{(\mu+\vartheta+1+\nu n)}{r}-\alpha, \tau+v\right),\left(b_{q}, B_{q}\right)\right] \\
& \cdot \frac{1}{(s+\vartheta+\nu n-1)} .
\end{aligned}
$$

Proof. From (10) and (25), it gives

$$
\begin{aligned}
& M\left\{R_{x, r}^{\mu, \alpha}\left(t^{\vartheta-1} E_{\lambda, \beta}^{\gamma}\left(a t^{\nu}\right)\right)\right\}(s)=\sum_{n=0}^{\infty} \frac{(\gamma)_{n}}{\Gamma(\lambda n+\beta) n !}\left(a^{n}\right) \\
& \quad \times H_{p+2, q+1}^{m, n+2}[k \mid \\
& \left.\quad\left(a_{p}, A_{p}\right),\left(1-\frac{(\mu+\vartheta+1+\nu n)}{r}, \tau\right),(-\alpha, v)\right] \\
& \left.\quad\left(-\frac{(\mu+\vartheta+1+\nu n)}{r}-\alpha, \tau+v\right),\left(b_{q}, B_{q}\right)\right] \\
& \quad M\left(t^{\vartheta+\nu n-1}\right) .
\end{aligned}
$$

Now, evaluating the Mellin transform of $t^{9+\nu n-1}$ using formula given by Mathai et al. [16]. we arrive at (26).

Theorem 6. All conditions follow from what is stated in Theorem 2 with $\mathfrak{R}(1-\vartheta)<1, \mathfrak{R}(s)>\mathfrak{R}(\nu)$; the following result holds:

$$
\begin{aligned}
M & \left\{K_{x, r}^{\varepsilon, \alpha}\left(t^{-\vartheta} E_{\lambda, \beta}^{\gamma}\left(a t^{-v}\right)\right)\right\}(s) \\
& =\sum_{n=0}^{\infty} \frac{(\gamma)_{n}}{\Gamma(\lambda n+\beta) n !}\left(a^{n}\right) \times H_{p+2, q+1}^{m, n+2}[k \mid \\
& \left.\left(a_{p}, A_{p}\right),\left(1-\frac{(\varepsilon+\vartheta+\nu n)}{r}, \tau\right),(-\alpha, v)\right] \\
& \left.\left(-\alpha-\frac{(\varepsilon+\vartheta+\nu n)}{r}, \tau+v\right),\left(b_{q}, B_{q}\right)\right] \\
& \frac{1}{(s-\varepsilon-\vartheta-\nu n)} .
\end{aligned}
$$

Proof. In similar manner, in proof of Theorem 5, we obtain the result (28). 
Laplace Transform (Sneddon [14]). The Laplace transform of a function $f(t)$, denoted by $F(s)$, is defined by the equation

$$
\begin{array}{r}
F(s)=(L f)(s)=L\{f(t) ; s\}=\int_{0}^{\infty} e^{-s t} f(t) d t \\
\Re(s)>0 .
\end{array}
$$

Provided the integral (29) is convergent and that the function, $f(t)$, is continuous for $t>0$ and of exponential order as $t \rightarrow$ $\infty,(29)$ may be symbolically written as

$$
\begin{aligned}
F(s) & =L\{f(t) ; s\} \\
\text { or } f(t) & =L^{-1}\{F(s) ; t\} .
\end{aligned}
$$

The following result is well known:

$$
\int_{0}^{\infty} e^{-s t} t^{p-1} d t=\frac{\Gamma(p)}{s^{p}}, \quad \mathfrak{R}(p)>1, \mathfrak{R}(s)>1 .
$$

Theorem 7. All conditions follow from what is stated in Theorem 1 with $\mathfrak{R}(s)>0$ and $\mathfrak{R}(\vartheta+\nu n)>0$; the following result holds:

$$
\begin{aligned}
& L\left\{R_{x, r}^{\mu, \alpha}\left(t^{\vartheta-1} E_{\lambda, \beta}^{\gamma}\left(a t^{v}\right)\right) ; s\right\} \\
& =s^{-\vartheta} \sum_{n=0}^{\infty} \frac{(\gamma)_{n}}{\Gamma(\lambda n+\beta) n !}\left(a s^{-\gamma}\right)^{n} \Gamma(\vartheta+\nu n) \\
& \quad \times H_{p+2, q+1}^{m, n+2}[k \mid \\
& \left.\quad\left(a_{p}, A_{p}\right),\left(1-\frac{(\mu+\vartheta+1+\nu n)}{r}, \tau\right),(-\alpha, v)\right] \\
& \left.\quad\left(-\frac{(\mu+\vartheta+1+\nu n)}{r}-\alpha, \tau+v\right),\left(b_{q}, B_{q}\right)\right]
\end{aligned}
$$

Proof. we can develop similar line by using result of Laplace integral (31).

Theorem 8. All conditions follow from what is stated in Theorem 2 with $\mathfrak{R}(s)>0$ and $\mathfrak{R}(1-\vartheta-v n)>0$; the following result holds:

$$
\begin{aligned}
L\{ & \left.K_{x, r}^{\varepsilon, \alpha}\left(t^{-\vartheta} E_{\lambda, \beta}^{\gamma}\left(a t^{-v}\right)\right)\right\}(s) \\
& =s^{1-\vartheta} \sum_{n=0}^{\infty} \frac{(\gamma)_{n}}{\Gamma(\lambda n+\beta) n !}\left(a s^{-\nu}\right)^{n} \Gamma(1-\vartheta-\nu n) \\
& \times H_{p+2, q+1}^{m, n+2}[k \mid \\
& \left.\left(a_{p}, A_{p}\right),\left(1-\frac{(\varepsilon+\vartheta+\nu n)}{r}, \tau\right),(-\alpha, v)\right] . \\
& \left.\left(-\alpha-\frac{(\varepsilon+\vartheta+\nu n)}{r}, \tau+v\right),\left(b_{q}, B_{q}\right)\right]
\end{aligned}
$$

Proof. In a similar manner, in proof of Theorem 7, we obtain the result (33).

Whittaker Transform (Whittaker and Watson [17]). Due to Whittaker transform, the following result holds:

$$
\begin{aligned}
\int_{0}^{\infty} e^{-t / 2} t^{\zeta-1} W_{\chi, \omega}(t) d t \\
=\frac{\Gamma(1 / 2+\omega+\zeta) \Gamma(1 / 2-\omega+\zeta)}{\Gamma(1-\chi+\zeta)},
\end{aligned}
$$

where $\mathfrak{R}(\omega \pm \zeta)>-1 / 2$ and $W_{\chi, \omega}(t)$ is the Whittaker confluent hypergeometric function:

$$
\begin{aligned}
W_{\omega, \zeta}(z)= & \frac{\Gamma(-2 \omega)}{\Gamma(1 / 2-\chi-\omega)} M_{\chi, \omega}(z) \\
& +\frac{\Gamma(2 \omega)}{\Gamma(1 / 2+\chi+\omega)} M_{\chi,-\omega}(z),
\end{aligned}
$$

where $M_{\chi, \omega}(z)$ is defined by

$$
M_{\chi, \omega}(z)=z^{1 / 2+\omega} e_{1}^{-1 / 2 z} F_{1}\left(\frac{1}{2}+\omega-\chi ; 2 \omega+1 ; z\right) .
$$

Theorem 9. Following what is stated in Theorem 1 for conditions on parameters, with $\mathfrak{R}[\omega \pm(\vartheta+\zeta+\nu n-1)]>1 / 2$, then the following result holds:

$$
\begin{aligned}
& \int_{0}^{\infty} e^{-\varphi t / 2} t^{\zeta-1} W_{\chi, \omega}(\varphi t)\left\{R_{x, r}^{\mu, \alpha}\left(t^{\vartheta-1} E_{\lambda, \beta}^{\gamma}\left(a t^{\nu}\right)\right)\right\} d t \\
& =\varphi^{1-\vartheta-\zeta} \sum_{n=0}^{\infty} \frac{(\gamma)_{n}}{\Gamma(\lambda n+\beta) n !}\left(a \varphi^{-\nu}\right) \\
& \times \frac{\Gamma(\omega+\vartheta+\zeta+\nu n-1 / 2) \Gamma(\vartheta-\omega+\zeta+\nu n-1 / 2)}{\Gamma(\vartheta-\chi+\zeta+\nu n)} \\
& \times H_{p+2, q+1}^{m, n+2}[k \mid \\
& \left.\begin{array}{c}
\left(a_{p}, A_{p}\right),\left(1-\frac{(\mu+\vartheta+1+\nu n)}{r}, \tau\right),(-\alpha, v) \\
\left(-\frac{(\mu+\vartheta+1+\nu n)}{r}-\alpha, \tau+v\right),\left(b_{q}, B_{q}\right)
\end{array}\right] .
\end{aligned}
$$

Proof. Using (10) and (34), it gives

$$
\int_{0}^{\infty} e^{-\varphi t / 2} t^{\zeta-1} W_{\chi, \omega}(\varphi t)\left\{R_{x, r}^{\mu, \alpha}\left(t^{\vartheta-1} E_{\lambda, \beta}^{\gamma}\left(a t^{\nu}\right)\right)\right\} d t
$$




$$
\begin{aligned}
& =\sum_{n=0}^{\infty} \frac{(\gamma)_{n}(a)^{n}}{\Gamma(\lambda n+\beta) n !} \times H_{p+2, q+1}^{m, n+2}[k \mid \\
& \left.\left(a_{p}, A_{p}\right),\left(1-\frac{(\mu+\vartheta+1+\nu n)}{r}, \tau\right),(-\alpha, v)\right] \\
& \left.\left(-\frac{(\mu+\vartheta+1+\nu n)}{r}-\alpha, \tau+v\right),\left(b_{q}, B_{q}\right)\right] \\
& \times \int_{0}^{\infty} e^{-\varphi t / 2} t^{(9+\zeta+\nu n-1)-1} W_{\chi, \omega}(\varphi t) d t .
\end{aligned}
$$

Assume that $t=k, \Rightarrow d t=d k / \varphi$; we get

$$
\begin{aligned}
= & \sum_{n=0}^{\infty} \frac{(\gamma)_{n}(a)^{n}}{\Gamma(\lambda n+\beta) n !} H_{p+2, q+1}^{m, n+2}[k \mid \\
& \left.\left(a_{p}, A_{p}\right),\left(1-\frac{(\mu+\vartheta+1+\nu n)}{r}, \tau\right),(-\alpha, v)\right] \\
& \left.\left(-\frac{(\mu+\vartheta+1+\nu n)}{r}-\alpha, \tau+v\right),\left(b_{q}, B_{q}\right)\right] \\
& \times \varphi^{1-\vartheta-\zeta-v n} \int_{0}^{\infty} e^{-k / 2} k^{(\vartheta+\zeta+v n-1)-1} W_{\chi, \omega}(k) d k .
\end{aligned}
$$

Interpreting the right-hand side of (39), using (34), we arrive at the result (37).

Theorem 10. Following what is stated in Theorem 2 for conditions on parameters, with $\mathfrak{R}[\omega \pm(-\vartheta+\zeta-v n-1)]>1 / 2$, then the following result holds:

$$
\begin{aligned}
\int_{0}^{\infty} & e^{-\varphi t / 2} t^{\zeta-1} W_{\chi, \omega}(\varphi t)\left\{K_{x, r}^{\varepsilon, \alpha}\left(t^{-\vartheta} E_{\lambda, \beta}^{\gamma}\left(a t^{-\nu}\right)\right)\right\} d t \\
& =\varphi^{\vartheta-\zeta} \sum_{n=0}^{\infty} \frac{(\gamma)_{n}}{\Gamma(\lambda n+\beta) n !}\left(a \varphi^{\nu}\right) \\
& \times \frac{\Gamma(\omega-\vartheta+\zeta-\nu n+1 / 2) \Gamma(-\vartheta-\omega+\zeta-\nu n+1 / 2)}{\Gamma(1-\vartheta-\chi+\zeta-\nu n)} \\
& \times H_{p+2, q+1}^{m, n+2}[k \mid \\
& \left.\left(a_{p}, A_{p}\right),\left(1-\frac{(\varepsilon+\vartheta+\nu n)}{r}, \tau\right),(-\alpha, v)\right] . \\
& \left.\left(-\alpha-\frac{(\varepsilon+\vartheta+\nu n)}{r}, \tau+v\right),\left(b_{q}, B_{q}\right)\right]
\end{aligned}
$$

Proof. In a similar manner, in proof of Theorem 9, we obtain the result (40).
K-Transform (Erdélyi et al. [18]). This transform is defined by the following integral equation:

$$
\begin{aligned}
\mathfrak{R}_{v}[f(x) ; p] & =g[p ; v] \\
& =\int_{0}^{\infty}(p x)^{1 / 2} K_{v}(p x) f(x) d x,
\end{aligned}
$$

where $\mathfrak{R}(p)>0 ; K_{v}(x)$ is the Bessel function of the second kind defined by ([18], p. 332)

$$
K_{v}(z)=\left(\frac{\pi}{2 z}\right)^{1 / 2} W_{0, v}(2 z)
$$

where $W_{0, v}(\cdot)$ is the Whittaker function defined in Erdélyi et al. [18].

The following result given in Mathai et al. ([16], p. 54, eq. 2.37) will be used in evaluating the integrals:

$$
\begin{aligned}
\int_{0}^{\infty} t^{\rho-1} K_{v}(a x) d x=2^{\rho-2} a^{-\rho} \Gamma\left(\frac{\rho \pm v}{2}\right) ; \\
\mathfrak{R}(a)>0 ; \mathfrak{R}(\rho \pm v)>0 .
\end{aligned}
$$

Theorem 11. Following what is stated in Theorem 1 for conditions on parameters, with $\mathfrak{R}(\omega)>0 ; \mathfrak{R}((\rho+\vartheta+\nu n-1) \pm \ell)>0$, then the following result holds:

$$
\begin{aligned}
\int_{0}^{\infty} & t^{\rho-1} K_{\ell}(\omega t)\left\{R_{x, r}^{\mu, \alpha}\left(t^{\vartheta-1} E_{\lambda, \beta}^{\gamma}\left(a t^{\nu}\right)\right)\right\} d t \\
= & 2^{\rho+\vartheta-3} \omega^{(1-\rho-\vartheta)} \sum_{n=0}^{\infty} \frac{(\gamma)_{n}}{\Gamma(\lambda n+\beta) n !}\left(a\left(\frac{2}{\omega}\right)^{\nu}\right) \\
\cdot & \Gamma\left(\frac{(\rho+\vartheta+\nu n-1) \pm \ell}{2}\right) \times H_{p+2, q+1}^{m, n+2}[k \mid \\
& \left.\left(a_{p}, A_{p}\right),\left(1-\frac{(\mu+\vartheta+1+\nu n)}{r}, \tau\right),(-\alpha, v)\right] . \\
& \left.\left(-\frac{(\mu+\vartheta+1+\nu n)}{r}-\alpha, \tau+v\right),\left(b_{q}, B_{q}\right)\right]
\end{aligned}
$$

Proof. Using (10) and (44), it gives

$$
\begin{aligned}
& \int_{0}^{\infty} t^{\rho-1} K_{\ell}(\omega t)\left\{R_{x, r}^{\mu, \alpha}\left(t_{p}^{\vartheta-1} \underset{p, \xi, \gamma}{K_{q}}\left(a t^{\nu}\right)\right)\right\} d t \\
& =\sum_{n=0}^{\infty} \frac{(\gamma)_{n}(a)^{n}}{\Gamma(\lambda n+\beta) n !} \times H_{p+2, q+1}^{m, n+2}[k \mid \\
& \left(a_{p}, A_{p}\right),\left(1-\frac{(\mu+\vartheta+1+\nu n)}{r}, \tau\right),(-\alpha, v) \\
& \quad\left(-\frac{(\mu+\vartheta+1+\nu n)}{r}-\alpha, \tau+v\right),\left(b_{q}, B_{q}\right) \\
& \quad \times \int_{0}^{\infty} t^{(\rho+\vartheta+\nu n-1)-1} K_{\ell}(\omega t) d t,
\end{aligned}
$$


and we get

$$
\begin{aligned}
= & \sum_{n=0}^{\infty} \frac{(\gamma)_{n}(a)^{n}}{\Gamma(\lambda n+\beta) n !} H_{p+2, q+1}^{m, n+2}[k \mid \\
& \left.\left(a_{p}, A_{p}\right),\left(1-\frac{(\mu+\vartheta+1+\nu n)}{r}, \tau\right),(-\alpha, v)\right] \\
& \left.\left(-\frac{(\mu+\vartheta+1+\nu n)}{r}-\alpha, \tau+v\right),\left(b_{q}, B_{q}\right)\right] \\
& \times 2^{\rho+\vartheta+\nu n-3} \omega^{(1-\rho-\vartheta-\nu n)} \Gamma\left(\frac{(\rho+\vartheta+\nu n-1) \pm \ell}{2}\right) .
\end{aligned}
$$

Interpreting the right-hand side of (46), we arrive at the result (44).

Theorem 12. Following what is stated in Theorem 2 for conditions on parameters, with $\mathfrak{R}(\omega)>0 ; \mathfrak{R}((\rho-\vartheta-v n) \pm \ell)>0$, then the following result holds:

$$
\begin{gathered}
\int_{0}^{\infty} t^{\rho-1} K_{\ell}(\omega t)\left\{K_{x, r}^{\varepsilon, \alpha}\left(t^{-\vartheta} E_{\lambda, \beta}^{\gamma}\left(a t^{-v}\right)\right)\right\} d t \\
=2^{\rho-\vartheta-2} \omega^{(\vartheta-\rho)} \sum_{n=0}^{\infty} \frac{(\gamma)_{n}}{\Gamma(\lambda n+\beta) n !}\left(a\left(\frac{\omega}{2}\right)^{\nu}\right) \\
\cdot \Gamma\left(\frac{(\rho-\vartheta-\nu n) \pm \ell}{2}\right) \times H_{p+2, q+1}^{m, n+2}[k \mid \\
\left.\left(a_{p}, A_{p}\right),\left(1-\frac{(\varepsilon+\vartheta+\nu n)}{r}, \tau\right),(-\alpha, v)\right] \\
\left.\left(-\alpha-\frac{(\varepsilon+\vartheta+\nu n)}{r}, \tau+v\right),\left(b_{q}, B_{q}\right)\right]
\end{gathered}
$$

Proof. In a similar manner, in proof of Theorem 11, we obtain the result (47).

\section{Properties of Integral Operators}

Here, we established some properties of the operators as consequences of Theorems 1 and 2. These properties show compositions of power function.

Theorem 13. Following all the conditions on parameters as stated in Theorem 1 with $\mathfrak{R}(\psi+9)>0$, then the following result holds true:

$$
\begin{aligned}
x^{\psi} & R_{x, r}^{\mu, \alpha}\left[t^{9-1} E_{\lambda, \beta}^{\gamma}(a t v)\right](x) \\
& =R_{x, r}^{\mu-\psi, \alpha}\left[t^{\psi+\vartheta-1} E_{\lambda, \beta}^{\gamma}\left(a t^{\nu}\right)\right](x) .
\end{aligned}
$$

Proof. From (10), the left-hand side of (48), we have

$$
\begin{aligned}
& x^{\psi} R_{x, r}^{\mu, \alpha}\left[t^{9-1} E_{\lambda, \beta}^{\gamma}\left(a t^{\nu}\right)\right](x) \\
& =\sum_{n=0}^{\infty} \frac{(\gamma)_{n}(a)^{n}}{\Gamma(\lambda n+\beta) n !} x^{9+\psi+\nu n-1} \times H_{p+2, q+1}^{m, n+2}[k \mid \\
& \left.\left(a_{p}, A_{p}\right),\left(1-\frac{(\mu+\vartheta+1+\nu n)}{r}, \tau\right),(-\alpha, v)\right] \\
& \left.\left(-\frac{(\mu+\vartheta+1+\nu n)}{r}-\alpha, \tau+v\right),\left(b_{q}, B_{q}\right)\right],
\end{aligned}
$$

and again, by (10), the right-hand side of (48) follows:

$$
\begin{aligned}
& R_{x, r}^{\mu-\psi, \alpha}\left[t^{\psi+\vartheta-1} E_{\lambda, \beta}^{\gamma}\left(a t^{\nu}\right)\right](x) \\
& =\sum_{n=0}^{\infty} \frac{(\gamma)_{n}(a)^{n}}{\Gamma(\lambda n+\beta) n !} x^{\vartheta+\psi+\nu n-1} \times H_{p+2, q+1}^{m, n+2}[k \mid \\
& \left.\left(a_{p}, A_{p}\right),\left(1-\frac{(\mu+\vartheta+1+\nu n)}{r}, \tau\right),(-\alpha, v)\right] \\
& \left.\quad\left(-\frac{(\mu+\vartheta+1+\nu n)}{r}-\alpha, \tau+v\right),\left(b_{q}, B_{q}\right)\right] .
\end{aligned}
$$

It seems that Theorem 13 readily follows due to (49) and (50).

Theorem 14. Following all the conditions on parameters as stated in Theorem 2 with $\mathfrak{R}(\beta+\vartheta)>0$, then the following result holds true:

$$
\begin{aligned}
& x^{-\psi} K_{x, r}^{\varepsilon, \alpha}\left[t^{-9} E_{\lambda, \beta}^{\gamma}\left(a t^{-v}\right)\right](x) \\
& =K_{x, r}^{\varepsilon-\psi, \alpha}\left[t^{-9-\psi} E_{\lambda, \beta}^{\gamma}\left(a t^{-\nu}\right)\right](x) .
\end{aligned}
$$

Proof. From (12), the left-hand side of (51), we have

$$
\begin{aligned}
& x^{-\psi} K_{x, r}^{\varepsilon, \alpha}\left[t^{-9} E_{\lambda, \beta}^{\gamma}\left(a t^{-\nu}\right)\right](x) \\
& =\sum_{n=0}^{\infty} \frac{(\gamma)_{n}(a)^{n}}{\Gamma(\lambda n+\beta) n !} x^{-\psi-\vartheta-\nu n} \times H_{p+2, q+1}^{m, n+2}[k \mid \\
& \left.\left(a_{p}, A_{p}\right),\left(1-\frac{(\varepsilon+\vartheta+\nu n)}{r}, \tau\right),(-\alpha, v)\right] \\
& \left.\left(-\alpha-\frac{(\varepsilon+\vartheta+\nu n)}{r}, \tau+v\right),\left(b_{q}, B_{q}\right)\right] .
\end{aligned}
$$


Again by (12), the right-hand side of (51) follows:

$$
\begin{aligned}
K_{x, r}^{\varepsilon-\psi, \alpha}\left[t^{-\vartheta-\psi} E_{\lambda, \beta}^{\gamma}\left(a t^{-\nu}\right)\right](x) & \\
= & \sum_{n=0}^{\infty} \frac{(\gamma)_{n}(a)^{n}}{\Gamma(\lambda n+\beta) n !} x^{-\psi-\vartheta-\nu n} \times H_{p+2, q+1}^{m, n+2}[k \mid \\
& \left.\left(a_{p}, A_{p}\right),\left(1-\frac{(\varepsilon+\vartheta+\nu n)}{r}, \tau\right),(-\alpha, v)\right] . \\
& \left.\left(-\alpha-\frac{(\varepsilon+\vartheta+\nu n)}{r}, \tau+v\right),\left(b_{q}, B_{q}\right)\right]
\end{aligned}
$$

It seems that Theorem 14 readily follows due to (52) and (53).

\section{Conclusions}

In this article, we have investigated and studied two classes of generalized fractional integral operators involving Fox's $H$-function as kernel due to Saxena and Kumbhat which are applied on M-L function. We discussed the actions of fractional integral operators under Euler, Mellin, Laplace, Whittaker, and $K$-transforms and results are given in better pragmatic series solutions. The majority of the results derived here are general in nature and compact forms are fairly helpful in deriving a variety of integral formulas in the theory of integral operators which arises in a range of problems of applied sciences like kinematics, diffusion equation, kinetic equation, fractal geometry, anomalous diffusion, propagation of seismic waves, turbulence, etc. We may obtain other special functions such as M-L function and Bessel-Maitland function (see, e.g., ([19-21]) as its special cases and, therefore, various unified fractional integral presentations can be obtained as special cases of our results.

\section{Data Availability}

No data were used to support this study.

\section{Conflicts of Interest}

The authors declare that they have no conflicts of interest regarding the publication of this paper.

\section{References}

[1] M. G. Mittag-Leffler, "Sur la nuovelle function $E_{\alpha}(x)$," Comptes rendus de l'Académie des Sciences, vol. 137, no. 2, pp. 554-558, 1903.

[2] A. Wiman, "Uber de Fundamental Satz in der Theorie derFunktionen $E_{\alpha}(x)$," Acta Mathematica, vol. 29, no. 1, pp. 191-201, 1905.

[3] T. R. Prabhakar, "A singular integral equation with a generalized Mittag Leffler function in the kernel," Yokohama Mathematical Journal, vol. 19, pp. 7-15, 1971.

[4] R. K. Saxena and R. K. Kumbhat, "Integral operators involving $H$-function," Indian Journal of Pure and Applied Mathematics, vol. 5, pp. 1-6, 1974.
[5] D. Baleanu, D. Kumar, and S. D. Purohit, "Generalized fractional integrals of product of two $H$-functions and a general class of polynomials," International Journal of Computer Mathematics, vol. 93, no. 8, pp. 1320-1329, 2016.

[6] V. Kiryakova, "On two Saigo's fractional integral operators in the class of univalent functions," Fractional Calculus and Applied Analysis, vol. 9, no. 2, pp. 159-176, 2006.

[7] K. S. Nisar, A. F. Eata, M. Al-Dhaifallah, and J. Choi, "Fractional calculus of generalized k-Mittag-Leffler function and its applications to statistical distribution," Advances in Difference Equations, vol. 304, p. 17, 2016.

[8] S. K. Ntouyas, S. D. Purohit, and J. Tariboon, "Certain Chebyshev type integral inequalities involving Hadamard's fractional operators," Abstract and Applied Analysis, Article ID 249091, p. 7, 2014.

[9] R. K. Saxena and M. Saigo, "Generalized fractional calculus of the $\mathrm{H}$-function associated with the Appell function F3," Journal of Fractional Calculus and Applications, vol. 19, pp. 89-104, 2001.

[10] H. M. Srivastava and Z. Tomovski, "Fractional calculus with an integral operator containing generalized Mittag-Leffler function in the kernel," Applied Mathematics and Computation, vol. 211, no. 1, pp. 110-198, 2009.

[11] C. Fox, "The $G$ and $H$ functions as symmetrical Fourier kernels," Transactions of the American Mathematical Society, vol. 98, pp. 395-429, 1961.

[12] A. M. Mathai and R. K. Saxena, The H-functions with Applications in Statistics and other Disciplines, John Wiley and Sons, 1974.

[13] H. M. Srivatava, K. C. Gupta, and S. P. Goyal, The H-Function of One and Two Variable With Applications, South Asian Publications, New Delhi, India, 1982.

[14] I. N. Sneddon, The Use of Integral Transform, Tata McGraw Hill, New Delhi, India, 1979.

[15] L. Debnath and D. Bhatta, Integral Transforms and Their Applications, Chapman and Hall (CRC Press), Taylor and Francis Group, London, UK, New york, NY, USA, 3rd edition, 2007.

[16] A. M. Mathai, R. K. Saxena, and H. J. Haubold, The H-function, Theory and Applications, Springer, New York, NY, USA, 2010.

[17] E. T. Whittaker and G. N. Watson, A Course of Modern Analysis, Cambridge University Press, Cambridge, UK, 1996.

[18] A. Erdélyi, W. Magnus, F. Oberhettinger, and F. G. Tricomi, Higher Transcendental Functions, vol. 2, McGraw-Hill, New York, NY, USA, 1954.

[19] V. N. Mishra, D. L. Suthar, S. D. Purohit, and H. M. Srivastava, "Marichev-Saigo-Maeda fractional calculus operators, Srivastava polynomials and generalized Mittag-Leffler function," Cogent Mathematics, vol. 4, pp. 1-11, 2017.

[20] S. D. Purohit, D. L. Suthar, and S. L. Kalla, "Some results on fractional calculus operators associated with the $M$-function," Hadronic Journal, vol. 33, no. 3, pp. 225-235, 2010.

[21] R. K. Saxena, J. Ram, and D. L. Suthar, "Fractional calculus of generalized Mittag-Leffler functions," Indian Academy of Mathematics, vol. 31, no. 1, pp. 165-172, 2009. 


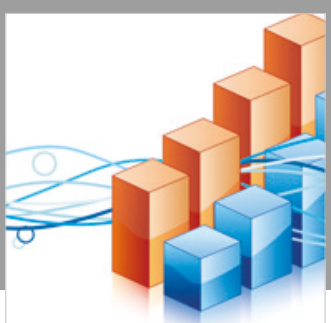

Advances in

Operations Research

\section{-n-m}
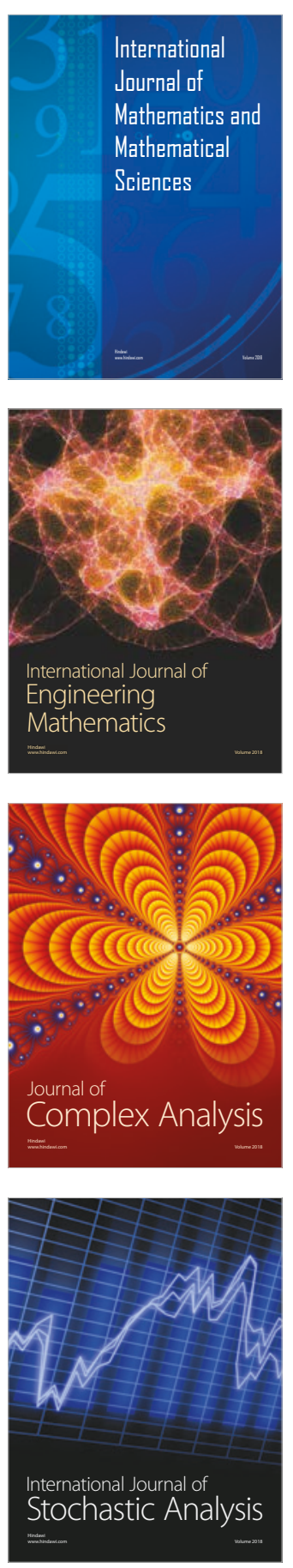


Journal of

Applied Mathematics
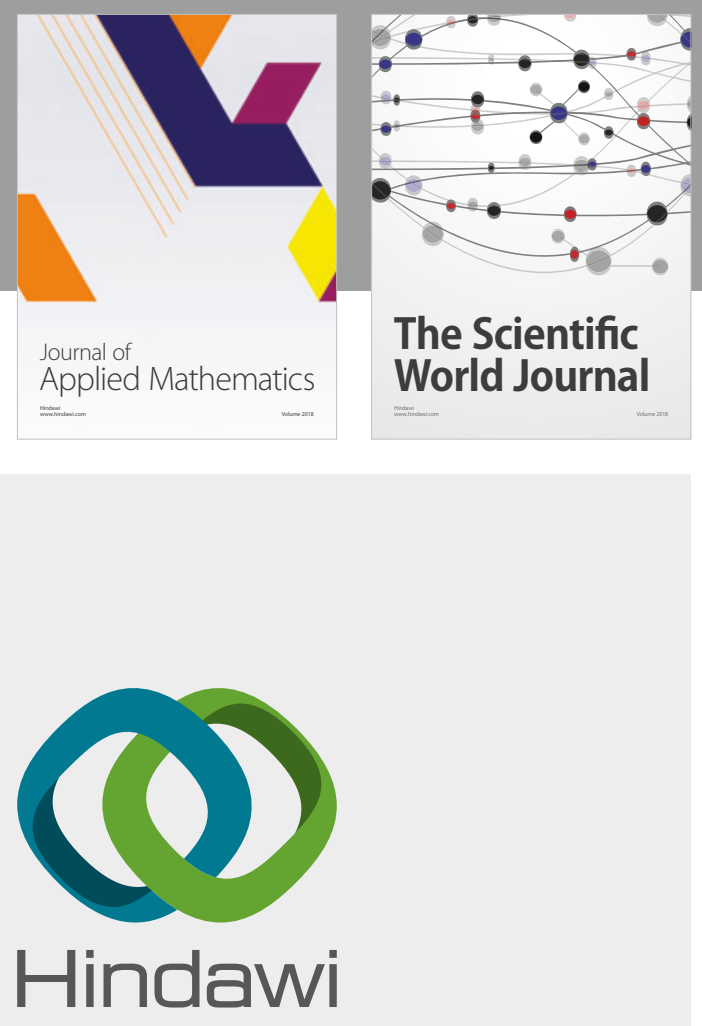

Submit your manuscripts at

www.hindawi.com

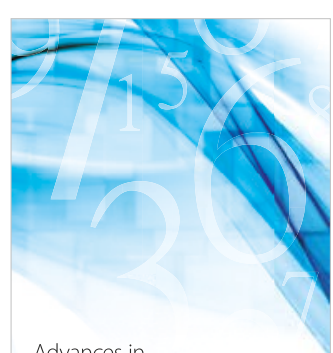

Advances in
Numerical Analysis
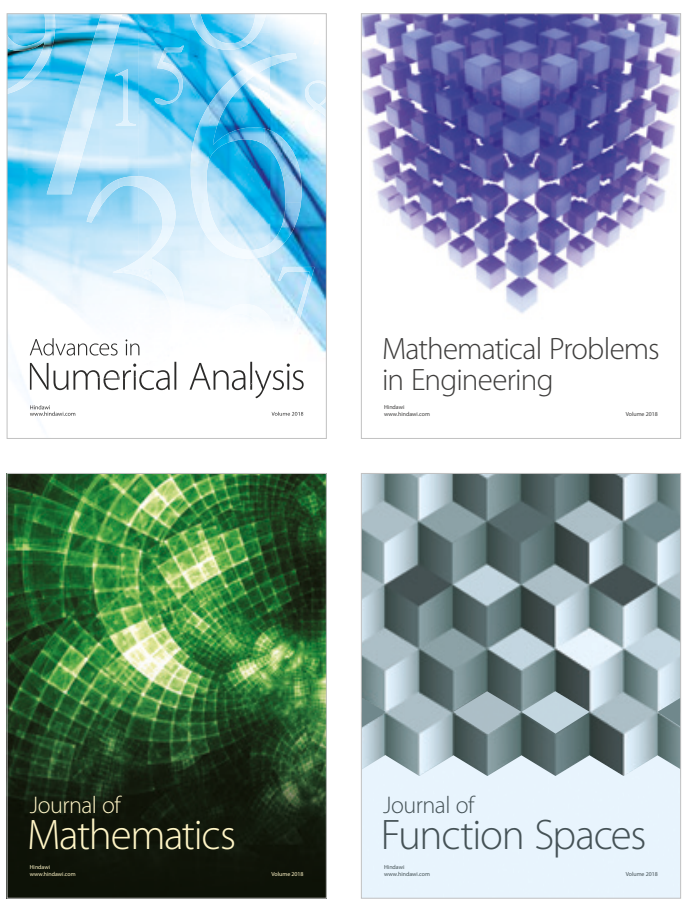

Mathematical Problems in Engineering

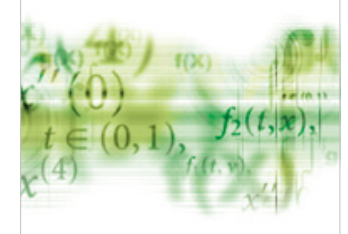

International Journal of

Differential Equations

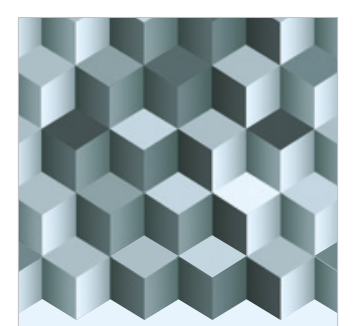

Journal of

Function Spaces

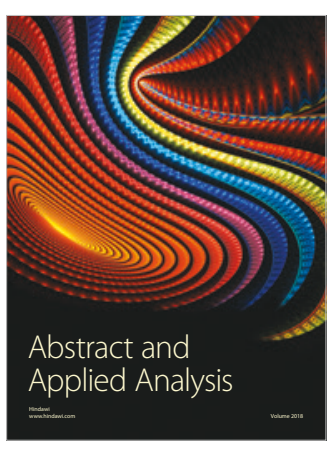

The Scientific

World Journal

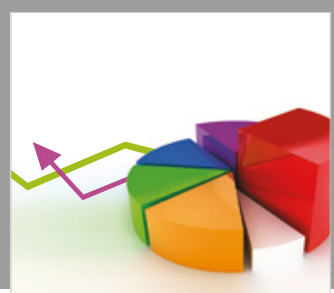

Journal of

Probability and Statistics
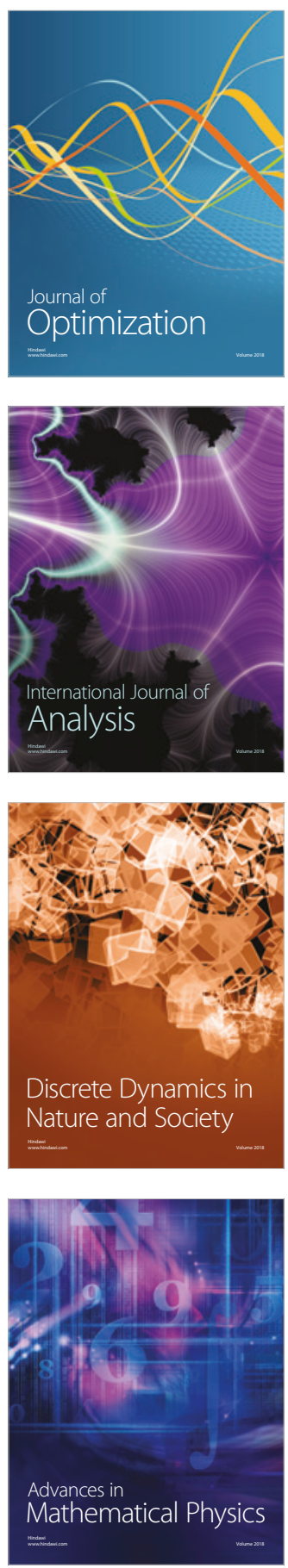